Troponin T Interpretation flow chart from Initial / Presentation Troponin Result (where eGFR is >40) NB must be more than one hour post onset of symptoms

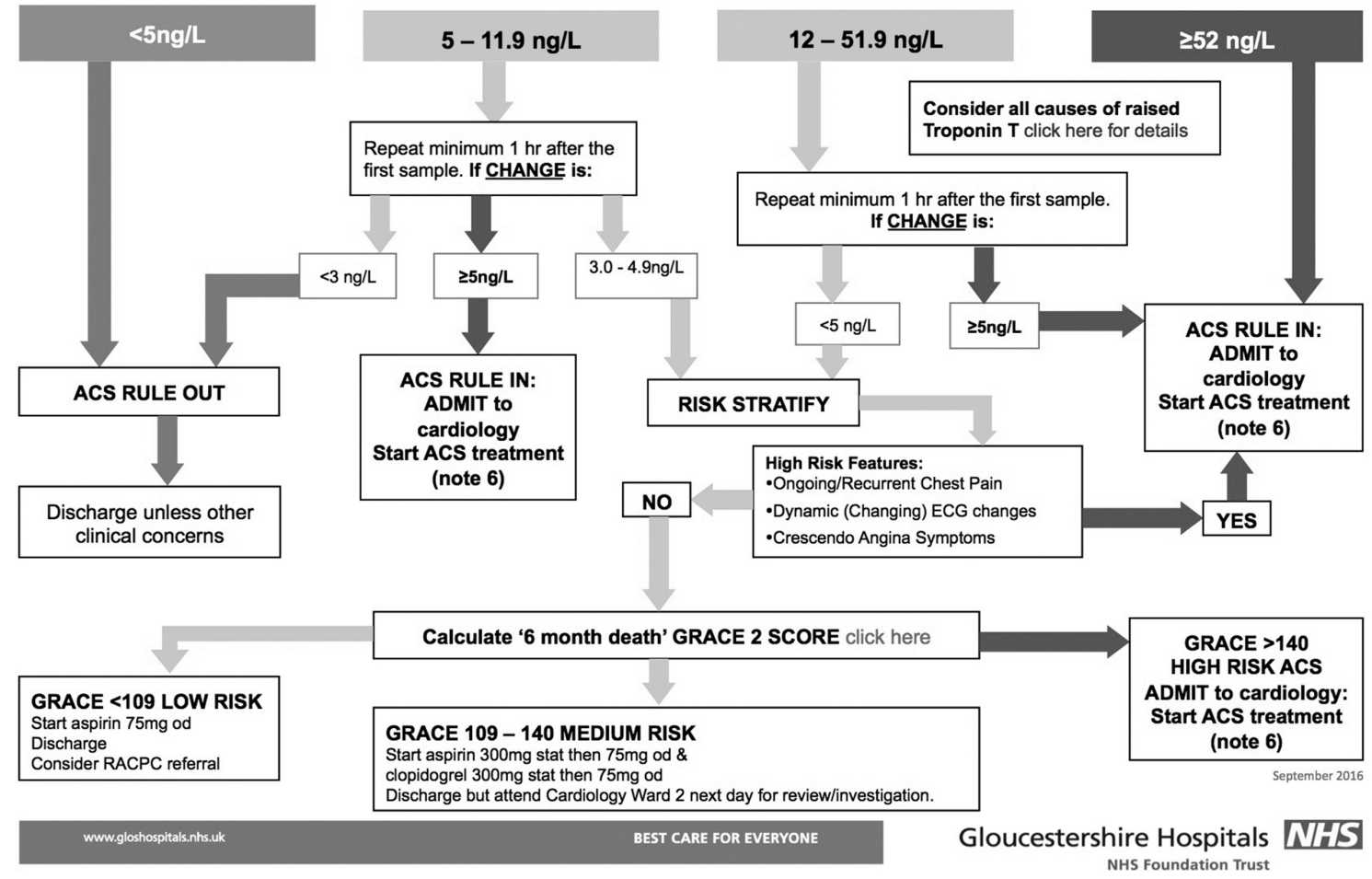

Abstract 67 Figure 1

measured at presentation, and if necessary a repeat test was performed at 1 hour. Patients were then stratified using the 1 hour Troponin pathway into low, medium and high risk groups.

All instances of hs-cTnT performed for 112 consecutive hours were included in analysis, 56 hours of the trial period and 56 hours of control data for comparison. We reviewed the cases to assess the performance and safety of the 1 hour troponin pathway using primary outcome data of discharge rates and subsequent myocardial infarction or death within 30 days.

Results 140 patients had a hs-cTnT performed. 51 cases did not present with chest pain or were admitted with a non-ACS diagnosis and were excluded from further analysis. During the trial period $21(54 \%)$ of patients were discharged directly home from the Emergency Department, compared with 15 (30\%) during the control period $(\mathrm{p}=0.02 ; n-1$ two-proportion $\left(\tilde{A} \square \hat{a} € i^{2}\right)$ analysis). There were no documented cases of subsequent myocardial infarction or death within 30 days follow up.

Conclusions There was a substantial increase in the percentage of patients discharged home from the Emergency Department during the 1 hour Troponin trial compared with the control period, demonstrating that this pathway facilitates rapid decision making and early discharge. Our estimate is that a further 3 admissions could have been avoided during the trial period if the pathway had been strictly implemented, and we predict that further education and familiarity for the Emergency Department staff will improve this. There were no cases of acute myocardial infarction or death in our cohort at 30 days, meaning that patient safety does not appear to be comprised.
To our knowledge this is the first trial of a 1 hour Troponin pathway in a UK DGH, and demonstrates that a 1 hour model can be applied to an unselected population and can safely reduce acute hospital admissions.

\section{THE IMPACT OF THE WEEKEND ADMISSION ON EARLY MORTALITY AFTER ACUTE CORONARY SYNDROME: A META-ANALYSIS OF OBSERVATIONAL STUDIES}

${ }^{1}$ Chun Shing Kwok*, ${ }^{2}$ Mohammed Al-Dokheal, ${ }^{2}$ Sami Aldaham, ${ }^{1}$ Claire Rushton, ${ }^{3}$ Robert Butler, ${ }^{4}$ Tim Kinnaird, ${ }^{5}$ Azfar Zaman, ${ }^{6} \mathrm{M}$ Justin Zaman, ${ }^{7}$ Adam Timmis, ${ }^{1}$ Mamas Mamas. ${ }^{1}$ Keele University; ${ }^{2}$ Al-Imam Mohammad Ibn Saud Islamic University; ${ }^{3}$ Royal Stoke University Hospital; ${ }^{4}$ University Hospital of UK; ${ }^{5}$ Freeman Hospital; ${ }^{6}$ James Paget University Hospital; ${ }^{7}$ Barts and London School of Medicine and Dentistry

\subsection{6/heartjnl-2017-311726.67}

Background A weekend effect where outcomes for patients admitted acutely during the weekend are worse than those for patients admitted during the week has been reported in many specialities across medicine. The evidence supporting a weekend effect among patients admitted with an acute coronary syndrome is conflicting. This systematic review and meta-analysis aims to determine if collectively there is a weekend effect in acute coronary syndrome.

Methods We searched Medline and EMBASE for cohort studies examining the association between weekend compared to weekday admission at any time of the day and early mortality (in-hospital or 30 day). Relevant studies were pooled using random effects meta-analysis for risk of early mortality. Additional analyses were performed considering only more recent studies (conducted after 2005) and by patient group (STEMI 
or NSTEMI) as well as meta-regression according to starting year and mean year of study.

Results A total of 26 studies were included with over 16 million participants incorporating 3.3 million weekend and over 12.5 million weekday admissions and the rates of mortality rates were $18.3 \%$ and $15.7 \%$, respectively. The pooled results of all 26 studies suggest that weekend admission was associated with a small increase in risk of early mortality (OR 1.05 95\% CI 1.03-1.07). The results for subgroups of STEMI and NSTEMI cohorts were not statistically significant and timing of admission after 2005 had minimal influence on the results (OR 1.06 95\% CI 0.95-1.17).

Conclusions There is a small weekend effect for admission with acute coronary syndrome that has persisted over time.

\section{DO HIGH SENSITIVITY TROPONIN ASSAYS ACTUALLY RESULT IN A SHORTER HOSPITAL STAY IN A REAL WORLD CLINICAL SETTING?}

${ }^{1}$ Arvind Singhal ${ }^{*}$, ${ }^{1}$ lain Parsons, ${ }^{2}$ Ed Nicol. ${ }^{1}$ Chelsea and Westminster Hospital; ${ }^{2}$ Royal Brompton Hospital

\subsection{6/heartjnl-2017-311726.68}

Background Hospitals face an immense bed pressure this winter. Admission to hospital for rule-out of MI is common, and early rule-out may expedite patient turnover.

Aims To assess the time to discharge following introduction of high sensitivity troponin I (hsTnI) in comparison to standard Troponin assays.

Methods A prospective cohort study was undertaken over a 3 month period following the introduction of hsTnI (ARCHITECT assay) at Chelsea and Westminster Hospital in London. Troponin assays were taken at presentation and after 3 hours. A positive result was defined as a high baseline troponin (>68 ng/L for males, $>30 \mathrm{ng} / \mathrm{L}$ for females) or a change of $>50 \%$ between the two samples with at least one sample above the 99th centile for gender. The assay results were matched to patient demographics, admission and discharge times and discharge diagnoses. All troponin testing other than that performed at admission were excluded. Results were compared against the conventional Troponin I assay (threshold of detection $32 \mathrm{ng} / \mathrm{L}$ ), with the 12 hour rule-out protocol, using 6 months of data prior to the introduction of the hsTnI assay. Results hsTnI were requested on 553 patients in the emergency department over a 3 month period (6.1 patients/day), compared with 647 patients who had conventional troponin I testing over a preceding 6 month period (3.6 patients/day).

The mean length of hospital stay was 3.83 days in the hsTnI group vs 2.92 days in the conventional troponin group $(p=0.04)$. The median length of stay was 0.82 days in both groups $(p=0.09)$. All patients who had 2 hsTnI requests ended up being admitted to hospital, even if both were undetectable. No patient who had an undetectable 1st troponin had a positive 2nd troponin. Overall 93/553 (17\%) hsTnI results were identified as positive, of which 33/553 (6\%) had acute coronary syndromes, with 22/553 (4\%) undergoing coronary intervention. 19/553 (3\%) had positive hsTnI from other cardiac causes 41/553 (7\%) had non-cardiac causes.

Conclusions The introduction of hsTnI has not resulted in a shorter length of hospital stay for patients undergoing troponin testing. Troponin testing became more frequent after the introduction of the new assay which may have resulted in extra, unnecessary admissions. Acute coronary syndromes were relatively uncommon. Combining early rule-out algorithms, such as discharging patients whose initial hsTnI measurement is undetectable, and more judicious use of troponin testing, may prevent unnecessary admissions.

Conflict of interests Nil

\section{CLINICAL OUTCOMES OF CLOPIDOGREL VERSUS TICAGRELOR IN THE ELDERLY PATIENTS WITH ACUTE CORONARY SYNDROME}

Lampson Fan, Asad Shabbir*, Stewart Mclure, Jocelyn Lam, Mark Cassar, Nicos Spyrou. Royal Berkshire Hospital

\subsection{6/heartjnl-2017-311726.69}

Background Acute coronary syndrome (ACS) remains one of the leading causes of death in the UK. One of the recent advances in treatment in the treatment of ACS is ticagrelor, a potent $\mathrm{P}_{2} \mathrm{Y}_{12}$ inhibitor, that has become the recommended anti-platelet by the ESC for the treatment of ACS due to its mortality benefit over clopidogrel. The increased anti-platelet inhibition however, also results in significantly increased nonCABG related bleeding. With the ageing population there is a rising proportion of elderly patient $(>75)$ presenting with ACS. The elderly patients generally have greater co-morbidities, higher risk of recurrent ischaemia and also higher risk of bleeding but there is a distinct lack of evidence for this cohort of patients. In the landmark PLATO study for example, only $15 \%$ of patients were over the age of 75 . It is unclear currently whether ticagrelor is beneficial in these elderly patients. Methods A retrospective cohort study was performed in patients aged 75 and over who were admitted to the Royal Berkshire Hospital (RBH) between 2013 and 2015 with acute coronary syndrome. Patients were discharged either on clopidogrel (147 patients) or ticagrelor (154 patients) according to the local trust protocol at the time. Clinical characteristics and 12 month clinical outcomes (all-cause mortality, cardiovascular deaths, bleeding, angina, STEMI, NSTEMI, dyspnoea and ischaemic stroke) were analysed. Crusade score was used to calculate the bleeding risk.

Results The age and baseline clinical characteristics are similar between the two groups. Both had moderate bleeding risk with mean Crusade score of 36.9 for the clopidogrel group and 37 for the ticagrelor group. There was no significant difference in all-cause mortality $(8 \%$ vs $10 \%, \mathrm{p}=0.55)$, cardiovascular mortality $(2.7 \%$ vs $1.9 \%, \mathrm{p}=0.72)$, ischaemic stroke $(0.6 \%$ vs $1.9 \%, p=0.62)$, admissions with non-cardiac chest pain $(5.2 \%$ vs $6.1 \%, \mathrm{p}=0.8)$, angina $(6.1 \%$ vs $5.2 \%, \mathrm{p}=0.8)$ or STEMI ( $2 \%$ vs $1.3 \%, \mathrm{p}=0.67)$ between patients discharged on clopidogrel or ticagrelor. Patients on clopidogrel however had significant increased re-admissions with NSTEMI compared to ticagrelor $(7.5 \%$ vs $1.9 \%, p=0.028)$. No increase in either major ( 8.4 vs $8.1 \%, \mathrm{p}=1)$ or minor bleeding $(20 \%$ vs $17 \%, p=1$ ) was observed in the ticagrelor compared to the clopidogrel group using TIMI-bleeding criteria. Only 1 patient reported significant dyspnoea in the ticagrelor group.

Conclusions Elderly patients discharged on ticagrelor had reduced re-admissions with NSTEMI without an increase in either major or minor bleeding compared to patients discharged on clopidogrel. 